Bull. Mater. Sci., Vol. 5. No. 2, June 1983, pp. 147-152. (C) Printed in India

\title{
Dispersion of photoelastic constants in doped KDP crystals
}

\author{
K V RAMANAIAH and K B R VARMA \\ Department of Physics, PSG College of Technology, Coimbatore 641 004, India
}

MS received 17 May 1982; revised 9 August 1982

\begin{abstract}
The photoelastic behaviour of KDP crystals with additives like borax, ADP, nickel phosphate, manganese phosphate and sodium dihydrogen orthophosphate has been studied as a variable birefringent compensator. The dispersion of the stress birefringence with wavelength was studied for a single orientation.
\end{abstract}

Keywords. Photoelastic studies; potassium dihydrogen orthophosphate; stress birefringence additives.

\section{Introduction}

Photoelastic and electrooptic effects find innumerable applications in industry such as light modulators and deflectors in laser communication. Among the electrooptic materials, crystals of the type potassium dihydrogen orthophosphate (KDP) are important because they are relatively easy to grow in the required size with good optical quality. However, no attempts have been made to improve the growth characteristics, electrooptic and photoelastic properties of these materials. Brice (1973) reported that borax $\left(\mathrm{Na}_{2} \mathrm{~B}_{4} \mathrm{O}_{7} 10 \mathrm{H}_{2} \mathrm{O}\right)$ could be used to increase the growth rate of these crystals, but no further studies have been reported. It is also not known as to how the addition of borax affects the electrooptic and photoelastic properties of these crystals. The solubilities, growth rates and electrooptic properties of boraxadded KDP crystals were studied earlier (Varma and Ramanaiah 1981). In the present paper the effect of the addition of borax and other dopants such as nickel phosphate $\left(\mathrm{NiPO}_{4}\right)$, ammonium dihydrogen orthophosphate (ADP), manganese phosphate $\left(\mathrm{Mn}_{3}\left(\mathrm{PO}_{4}\right)_{2}\right)$ and sodium dihydrogen orthophosphate $\left(\mathrm{NaH}_{2} \mathrm{PO}_{4}\right)$ on the photoelastic properties of KDP crystals is reported.

The KDP crystals with various concentrations of borax (mole fraction), $\mathrm{NiPO}_{4}$, ADP, $\mathrm{Mn}_{3}\left(\mathrm{PO}_{4}\right)_{2}$, and $\mathrm{NaH}_{2} \mathrm{PO}_{4}$ (by weight \%) were grown at room temperature by Holden's rotary crystallizer technique. The crystals thus grown were less hygroscopic, optically clear, fairly large in sizes and free from strains. The presence of additives like borax and dopants in the grown crystals was confirmed by subjecting them to chemical and $x$-ray anadysis and these studies were also reported earlier (Ramanaiah and Varma 1982). 


\section{Photoelastic dispersion}

\subsection{Experimental arrangement}

The experimental technique used in this study is the same as that developed by Narasimhamurthy and Veerabhadra Rao (1968). This consists of white light rendered parallel by a collimating lens, passes through a polarizer whose vibration direction is inclined at $45^{\circ}$ to the vertical. Light enters the crystal, the birefringent compensator (BC) and then a soleil babinet compensator (SBC) (with the eye piece removed). (BC) is placed with one of its axes vertical and the other two axes horizontal on a stand which in turn can be rotated about its vertical axis. The analyser consists of a polaroid with its vibration direction perpendicular to that of the polarizer. The emergent beam of light is focussed on the slit of a constant deviation spectrometer (CDS) by a condensing lens. A diaphragm is used to cut out the stray light. The experimental crystal is stressed by introducing it between two knife edges of a stressing device, as shown in figure 1.

\subsection{Procedure}

Initially, SBC is calibrated for different wavelengths of light. Fringe width at any wavelength is the distance through which the micrometer screw of SBC has to be moved, so that the fringe of any particular order at that wavelength in the channelled spectrum $C$ in the spectrometer, is replaced by the next order fringe at the same wavelength. A graph plotted between the wavelength and the fringe width to obtain the smoothened values for fringe widths at various wavelengths, gives a straight line over the range of wavelength.

A birefringent crystal plate similar to the experimental one, was placed immediately after the experimental crystal and was oriented to get three or four fringes in the visible region of the spectrum. The experimental crystal was slightly stressed by a mechanical lever arrangement to see the direction of the fringe movement. One of the fringes was brought to $6000 \AA$ by working on the SBC. After checkins the uniformity of the stress distribution in the crystal, the photoelastic birefringence was linearised for three different loads. An approximate value of the shift in terms

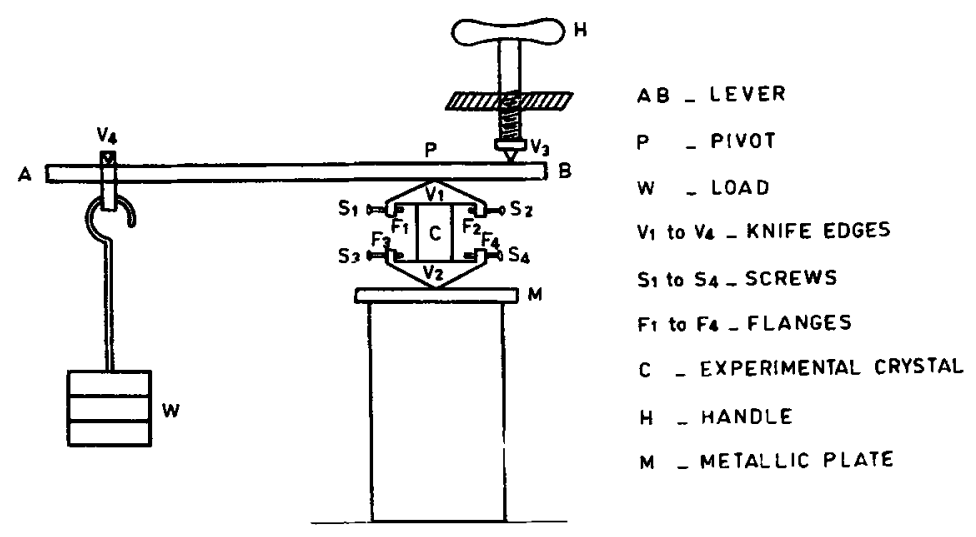

Figure 1. Mechanical device to stress the crystal. 
of SBC divisions was determined for a known load and a specified mechanical advantage at $6000 \AA$. The SBC was brought back to the zero order position from the same side as above. To study the dispersion of the photoelastic constant, the wavelength of each of the fringes in the spectrum was repeatedly recorded and a known load applied to the crystal. The new position $\lambda^{\prime}$. of each of the fringes in the spectrum was noted. The micrometer screw of the SBC was rotated in the proper direction, through the same number of divisions as determined earlier to compensate the stress birefringence. Finally the wavelength $\lambda^{\prime \prime}$ corresponding to each of the fringes was again recorded. From the above three sets of readings for each fringe, the shift $\Delta \lambda^{\prime}\left(\lambda \sim \lambda^{\prime}\right)$ due to the stress on the crystal and $\Delta \lambda^{\prime \prime}\left(\lambda^{\prime \prime} \sim \lambda^{\prime}\right)$ due to the movement of wedges of the SBC were determined. A graph was drawn between the wavelength and the shifts due to the loaded crystal $\left(\Delta \lambda^{\prime}\right)$ and that due to Soleil-Babinet compensator $\left(\Delta \lambda^{\prime \prime}\right)$. From these smoothened values of $\Delta \lambda^{\prime}$ and $\Delta \lambda^{\prime \prime}$, the load and the corresponding number of divisions required to move the fringe through unit $\AA$ were obtained. The values of $C_{\lambda}$ were calculated using the formula

$$
C_{\lambda}=\frac{\Delta \lambda^{\prime}}{\Delta \lambda^{\prime \prime}} \frac{x \lambda b}{f_{\lambda} m M 1000 g}
$$

where $x$ is the number of divisions moved in SBC, $b$ the breadth of the crystal, $f_{\lambda}$ the constant of the SBC for that particular wavelength, $M$ the load applied, $m$ the mechanical advantage and $g$ the gravitational force. The appropriate dimensions of the crystals were substituted in the above formula to determine $C_{\lambda}$.

\section{Photoelastic effect in KDP crystals}

\subsection{Pure KDP}

Initially, the experiments were carried out on pure KDP crystals to standardize the experimental set-up and to compare the results of KDP with additives and dopants. The KDP crystal was stressed along [001] and the consequent optical path retardation along [001] was measured at room temperature $\left(29^{\circ} \mathrm{C}\right)$ at various wavelengths. The photoelastic constants calculated are given in table 1 . The dispersion of photoelastic constant $C_{\lambda}$ with wavelength is shown in figure 2 . The trend in the variation of $C_{\lambda}$ with wavelength is similar to that reported earlier (Veerabhadra Rao 1972) and the results obtained for KDP agree with those reported earlier.

\section{$3.2 \mathrm{KDP}$ with borax}

The photoelastic constants calculated for KDP with $0.05,0.075$ and $0.1 \mathrm{M}$ borax at various wavelengths (figure 2) show that the photoelastic constant decreases with increasing wavelengths. KDP with borax showed higher values of photoelastic constants as compared to those of pure KDP. Among KDP crystals with borax, KDP with $0.075 \mathrm{M}$ borax has higher photoelastic constant values than those with 0.05 and $0.1 \mathrm{M}$ borax. Our results indicate that KDP crystals with borax are photoelastically soft probably due to the optimum value of borax. 


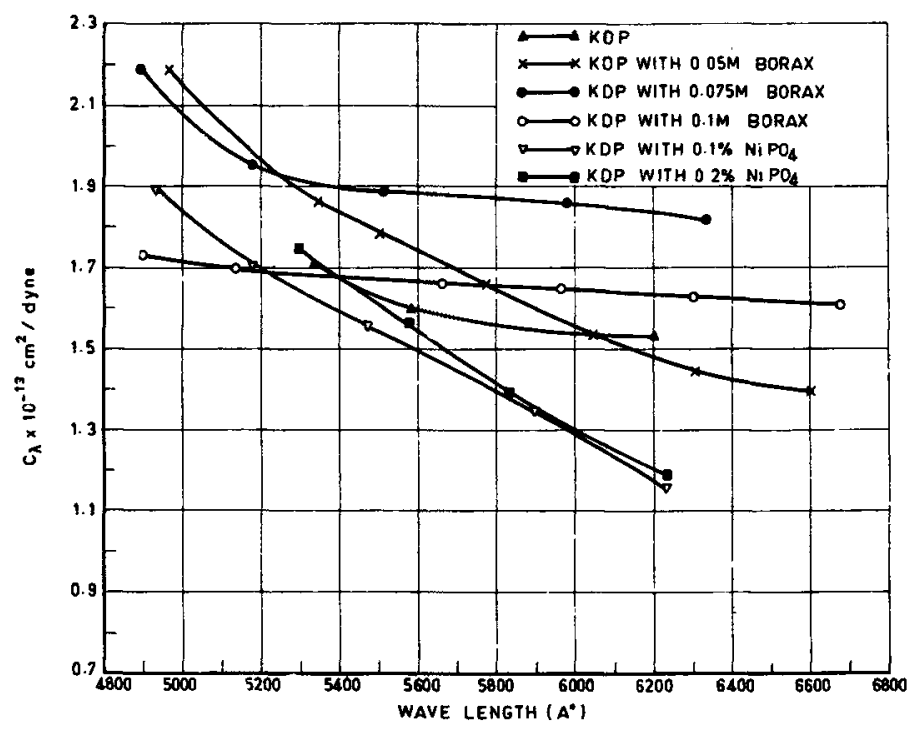

Figure 2. Dispersion of Brewster's constant $C_{\lambda}$ with wavelength for doped KDP crystals.

Table 1. Dispersion of Brewster's Constant $C_{\lambda}$ of KDP

\begin{tabular}{|c|c|c|c|c|}
\hline \multirow{3}{*}{$\begin{array}{l}\text { Initial position } \\
\text { of the fringe }(\AA)\end{array}$} & \multicolumn{2}{|c|}{$\begin{array}{l}\text { Shift of the fringe } \\
(\AA) \text { due to }\end{array}$} & \multicolumn{2}{|c|}{$\begin{array}{l}\text { Brewster's } \\
\text { constant } C_{\lambda}\end{array}$} \\
\hline & \multirow{2}{*}{$\begin{array}{l}\text { Stress on the } \\
\text { crystal }\end{array}$} & \multirow{2}{*}{$\begin{array}{c}\text { Compensation } \\
\text { by SBC }\end{array}$} & \multicolumn{2}{|c|}{$-C_{\lambda} \times 10^{-13} \mathrm{~cm}^{2} /$ dyne } \\
\hline & & & Present study & Literature \\
\hline 5330 & 12 & 13 & 1.711 & 1.748 \\
\hline 5580 & 13 & 15 & 1.603 & 1.745 \\
\hline 5885 & 14 & 16 & 1.604 & 1.694 \\
\hline 6200 & 16 & 19 & 1.539 & 1.635 \\
\hline
\end{tabular}

Stress along [001]; Observation along [100]; Change of load on the crystal $=1 \times 6.9577 \mathrm{~kg}$; No. of divisions of $\mathrm{SBC}$ rotated $=25$.

\subsection{KDP with nickel phosphate}

The photoelastic constants calculated at various wavelengths of light for KDP crystals with 0.1 and $0.2 \%$ nickel phosphate are shown in figure 2. It is seen that KDP crystals have low values of photoelastic constants as compared to pure KDP crystals. The presence of nickel ions in KDP perhaps makes it photoelastically hard.

\section{$3.4 K D P$ with $A D P$}

The photoelastic constants were calculated for KDP with $1 \%$ ADP and KDP with $3 \%$ ADP for various wavelengths. The dispersion of photoelastic constant $C_{\lambda}$ with 


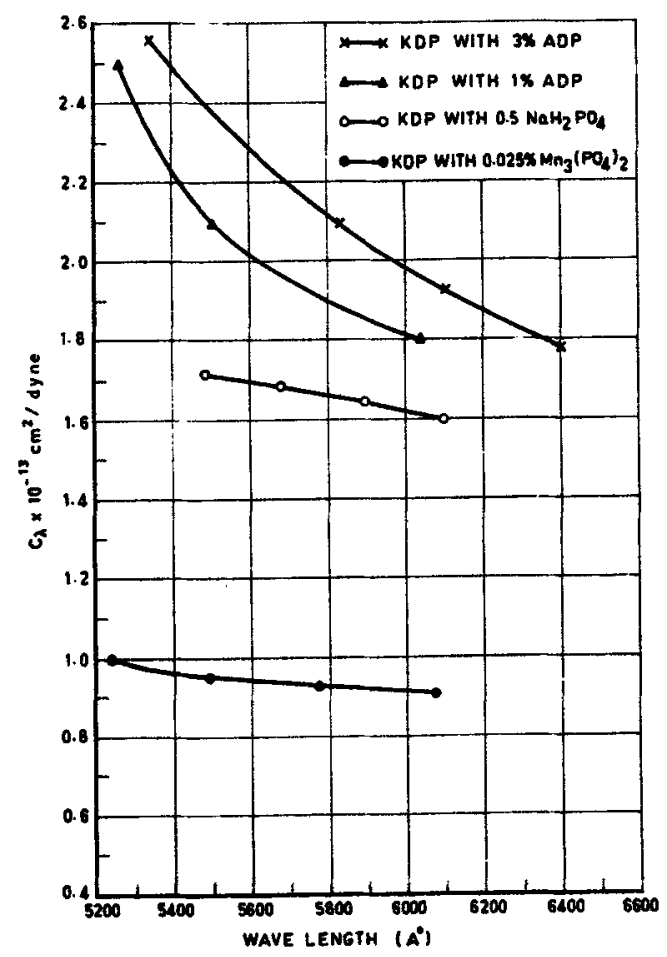

Figure 3. Dispersion of Brewster's constant $C_{\lambda}$ with wavelength for doped KDP crystals.

wavelength for both the crystals is shown in figure 2. The photoelastic constants decrease with increase in wavelength for both the crystals. The photoelastic constants of KDP with $1 \%$ and $3 \%$ ADP are higher than those of pure KDP. Among the crystals of KDP with $1 \%$ and $3 \%$ ADP, the latter has higher values of photoelastic constants implying that they increase with increasing concentrations of ADP in KDP. Thus, it was confirmed that the presence of ammonium ions in KDP makes it photoelastically soft.

\section{$3.5 \mathrm{KDP}$ with $\mathrm{NaH}_{2} \mathrm{PO}_{4}$ and $\mathrm{Mn}_{3}\left(\mathrm{PO}_{4}\right)_{2}$}

The photoelastic constants determined at various wavelengths for KDP with $0.5 \%$ sodium dihydrogen orthophosphate and KDP with $0.025 \%$ manganese phosphate are represented graphically in figure 3. The trend in the dispersion of $C_{\lambda}$ with wavelength for these two crystals is the same as that of pure KDP and other crystals.

The crystals of KDP with $0.5 \%$ sodium dihydrogen orthophosphate and $0.025 \%$ manganese phosphate have low photoelastic constant values as compared to those of pure KDP. Among these two, KDP with $0.025 \%$ manganese phosphate has lower photoelastic constant values. This would mean that the presence of manganese ions in KDP crystals lowers the photoelastic constant values. 


\section{Conclusions}

The photoelastic constants of KDP crystals tend to decrease with the addition of dopants such as nickel phosphate, manganese phosphate and sodium dihydrogen orthophosphate. However, the results obtained for crystals like KDP with borax and KDP with ADP are significant, being higher than those of pure KDP.

\section{Acknowledgements}

The authors thank Prof. T S Narasimhamurthy for valuable discussions. One of them (KBRV) is thankful to the UGC for a fellowship.

\section{References}

Brice J C 1973 The growth of crystals from liquids (London: North Holland) 12286

Varma K B R and Ramanaiah K V 1981 Bull. Mater. Sci. 3 307-315

Ramanaiah K V and Varma K B R 1982 Indian J. Pure Appl. Phys.

Narasimhamurthy T S and Veerabhadra Rao K 1968 Indian J. Pure Appl. Phys. 6 367-370

Veerabhadra Rao K 1972 Photoelastic and linear electrooptic dispersion in some non cubic crystals; Ph.D. Thesis, Osmania University, Hyderabad, India 